\title{
Resource Allocation for Downlink Spectrum Sharing in Cognitive Radio Networks
}

\author{
Patrick Mitran, Long Le, Catherine Rosenberg, and André Girard
}

\begin{abstract}
We consider a resource allocation problem for spectrum sharing in cognitive radio networks. Specifically, we investigate the joint subchannel, rate and power allocation for secondary users which share, in a non-disruptive manner, some frequency bands with primary users using OFDM technology. We consider the resource allocation problem for downlink and take into account the maximum total power constraints of the base station and the power constraints determined by distributed spectrum sensing and scanning. We formulate a resource allocation problem as an optimization problem which achieves max-min rate sharing among users. We propose both integer program based optimal and suboptimal fast and low complexity approaches for the spectrum sharing problem. Numerical results are then presented for the proposed heuristics and compared with the optimal solution.
\end{abstract}

Index Terms-Cognitive radio, spectrum access, OFDM, IEEE 802.22.

\section{INTRODUCTION}

Current empirical measurements indicate that most licensed radio frequency bands are under-utilized. In particular, FCC measurements show that some licensed spectrum sees as little as $10 \%$ usage, leaving up to $90 \%$ unused [1]. A prime example of under-utilized spectrum is the TV broadcast band, where most channels are not occupied or only partially occupied by licensed devices.

Spurred by these observations, a working group was formed in late 2004 to develop a standard physical, MAC and air interface (designated as IEEE 802.22) for use by licenseexempt devices (called secondary users) organized in a star topology around a license-exempt base station (BS) on a non-interfering basis in the TV broadcast band to provide broadband service to rural areas. This technology is often referred to as cognitive radio, due to the sensing requirements that are necessary to a) avoid causing harmful interference to primary users (the licensees, also called primary users), and b) exploit unused spectrum. The TV broadcast band is also used by Private Land Mobile Radio Services (PLMRS)and Land Transportation Radio Services implying that the occupancy of a set of subchannels by a licensed user can vary relatively fast.

In this paper, we consider resource allocation problems for cognitive wireless regional area networks (WRAN) that are similar in topology to IEEE 802.22 networks but differ in that we allow secondary devices to use channels occupied by primary users as long as the interference they create remains under control. In this paper, we limit ourselves to the downlink only.

We consider a network of $N$ secondary users (denoted as $\mathrm{CPE}$ or consumer premise equipment) which communicate

P. Mitran, C. Rosenberg and Long Le are with the Department of Electrical and Computer Engineering, University of Waterloo, Canada. A. Girard is with GERAD, Groupe d'Etudes et de Recherche en Analyse des Décisions, Montréal, Canada. directly with a base station over unlicensed frequency bands. We assume that, as in IEEE 802.22, the BS as well as the CPEs distributively sense their environment using specialized signature methods and fuse their collected data in order to determine the presence or absence of primary users in different channels or subchannels. In this paper, we assume that as a result of this distributed sensing, a vector $T$ is created, which provides the BS with constraints on its transmit power on any given channel or subchannel to avoid harmful interference to primary users. Thus this vector decouples the problem of spectrum sensing from that of resource allocation. In practice, $T$ will change with time due to the mobile nature of some of the primary users and thus, fast methods to allocate resources to the WRAN CPEs (the secondary users) are needed to avoid interference to primary users.

We also assume an OFDM-based system. This has the advantage that if narrowband incumbent users are detected, the secondary network can avoid creating harmful interference by limiting the transmit power on the appropriate subcarriers. In practice, OFDM systems partition subcarriers into groups called subchannels, which are the smallest unit that can be allocated for transmission to or reception from a CPE. Furthermore, no two CPEs may be assigned to the same subchannels. We assume that there are $M_{1}$ available channels and each channel is divided into $M_{2}$ subchannels for a total of $M=M_{1} M_{2}$ subchannels.

Since in practice, coding is applied on all subcarriers of a subchannel, for each subchannel, we associate a rate $R_{z}$ corresponding to a combined modulation and coding scheme $z$. The information transmitted on the subchannel is successfully received if the aggregate SNR (Signal to Noise Ratio) on the subchannel is above some threshold $\gamma_{z}$, for some appropriate notion of aggregate SNR.

We are interested in finding the joint subchannel, rate and power allocation for all $N$ CPEs (downlink only) such that some objective function is optimized. This allocation is static in that, it will remain unchanged until a new vector $T$ is generated and a new allocation is computed as a result of it.

The contributions of this paper are four-fold:

- We formulate a cognitive radio resource allocation problem for the downlink of a star network where the constraints due to the primary users are summarized in vector $T$,

- We propose two fast heuristic algorithms that solve the resource allocation problem,

- We propose a method to generate realistic test-cases,

- We evaluate our heuristics with respect to exact results and bounds obtained through cplex, a commercial integer linear program solver for realistic scenarios (several hundred subchannels, several tens of CPEs and several transmission schemes). 
The remaining of this paper is organized as follows. Section II provides background on resource allocation in traditional non-cognitive OFDM systems. In Section III, the downlink resource allocation problem is formalized mathematically while in Sections IV and V respectively numerical methods and results are presented. Finally, Section VI concludes this work.

\section{Resource Allocation for OFDM-BAsed WIRELESS NETWORKS}

Resource allocation in OFDM-based wireless networks has been an active research topic in the past several years. Generally the focus is on the downlink allocation problem, which is usually formulated as an optimization problem where a joint subcarrier, rate, and power allocation is considered.

For the downlink case, there are two important resource allocation problems. The first problem aims at minimizing the total transmission power while providing certain required transmission rates for different users [2], [3]. The second problem optimizes a given function of the transmission rates of the different users under a total power constraint at the BS [5]- [7]. These problems are referred respectively as margin adaptive (MA) and rate adaptive (RA) in the literature [5].

In [6], the problem of maximizing the total rate subject to a total power constraint at the BS and proportional rate sharing among different users was considered. Moreover, the problem of maximizing a total network utility under a total power constraint was considered in [7] where suboptimal algorithms under fixed or adaptive power allocation were developed.

The cognitive resource allocation problem investigated in this paper is fundamentally different from existing works in the literature for two reasons. The first one is due to the extra power constraints given by vector $T$. This new set of power constraints limits the transmit power on each allocated subchannel and renders the problem significantly more difficult. The second reason has to do with the rate of change of vector $T$ which forces us to develop very fast solutions. Indeed contrary to the OFDM scenario in which if we do not update the subchannel assignment too often we only incur a potential loss of efficiency, in cognitive radio systems we cannot afford to react too slowly to changes in $T$ since it would affect the primary users.

\section{PRoblem Formulation}

Formally, we consider a star network made of one BS and $N$ CPEs. There are $M$ subchannels and we only study the downlink (i.e., from the BS to the CPEs) allocation problem. The BS has a constraint on the maximum total power $\bar{P}_{\max }$ that it can use. The system is multi-rate, which means that a device can use any of $\bar{z}$ transmission modes on each allocated subchannel (each transmission mode corresponds to one particular modulation and coding scheme). Transmission mode $z$ yields a rate $R_{z}$ and requires an SNR threshold of $\gamma_{z}$ to achieve some desired block error rate. Given the noise level at the receiving side, we can calculate the minimum required transmit power $f_{i j}(z)$ from the BS to CPE $i$ on subchannel $j$ to use transmission mode $z$. This is a function of the distance between the BS and CPE $i$, the subchannel gains $g_{i j}$ (we do not assume that all the subchannel gains are identical) and any interference from primary transmissions to secondary users.

The BS has a vector $T$ that gives for each subchannel $c_{j}$ $(1 \leq j \leq M)$ the maximum power $\bar{P}_{j}$ that the BS could use to transmit over $c_{j}$ without causing harmful interference to any primary user ${ }^{1}$.

We are interested in finding the joint subchannel, rate, and power allocation for all $N$ CPEs such that some objective function is optimized. In the following, we will consider the objective function that maximizes the minimum aggregated rate offered to a CPE since we believe that it is the appropriate objective in a managed network.

Specifically, let us define the integer variable $s_{i j z}$ as follows: $s_{i j z}=1$ if subchannel $j$ is allocated to CPE $i$, i.e., the $\mathrm{BS}$ will transmit to $\mathrm{CPE} i$ on this subchannel using transmission mode $z(1 \leq z \leq \bar{z})$, otherwise $s_{i j z}=0$. A subchannel can only be allocated to one CPE $i$.

Then the optimization problem can be written as

$$
\begin{aligned}
& \max _{s_{i j z}} \min _{i} \sum_{j=1}^{M} \sum_{z=1}^{\bar{z}} R_{z} s_{i j z} \\
& \text { subject to } \\
& \quad \sum_{i=1}^{N} \sum_{z=1}^{\bar{z}} s_{i j z} \leq 1 \\
& f_{i j}(z) s_{i j z} \leq \bar{P}_{j} \\
& \sum_{i=1}^{N} \sum_{j=1}^{M} \sum_{z=1}^{\bar{z}} f_{i j}(z) s_{i j z} \leq \bar{P}_{\max }
\end{aligned}
$$

Eq. (2) says that a given subchannel cannot be allocated to more than one pair $(i, z)$. Eq. (3) refers to the power constraints as given by $T$ while (4) is the constraint on the total transmit power.

The problem (1)-(4) is an integer linear program whose inputs are: $N, M, \bar{z}, \bar{P}_{\max }, \bar{P}_{j}$ and the functions $f_{i j}(z)$. A typical WRAN would have tens of CPEs, up to several hundred of subchannels and several transmission modes. For example in a realistic scenario in which $N=40, M=120, \bar{z}=5$, the optimization problem would have 24,000 variables $s_{i j z}$ out of which only 120 are non-zero.

\section{ExACT AND Approximate Solutions}

\section{A. Exact Solutions}

The optimal allocation problem (1)-(4) is a hard combinatorial problem. Even though we cannot expect to solve it exactly for large instances, there is some information to be obtained from even a partial solution obtained from a branchand-bound algorithm. We have tried to solve a number of cases with cplex, all with a time limit of ten minutes on a Mac Pro server with two 3.0GHz Quad-Core Intel Xeon 5400 Series processors and 8 GB memory. The results varied widely depending on the actual data. In some cases, an exact solution was obtained within seconds or minutes, in other cases, the program ran to its time limit and in some other cases, it stopped because the enumeration tree became too large. The important point is that even in these cases, cplex provides us with a lower and an upper bound from which we can estimate the accuracy of a heuristic.

\footnotetext{
${ }^{1}$ Since subchannels are typically blocks of 30-60 consecutive subcarriers, we assume that sufficient subcarrier sidelobe rejection can be achieved so that the sidelobes of a subchannel do not create significant interference. If this is not the case, then the optimization problem must be reformulated based on the amount of rejection that can be achieved.
} 


\section{B. Heuristic 1: A Greedy Heuristic}

Given that the problem cannot be solved exactly and quickly for moderate to large problem sizes, there is an obvious need for approximate solutions. Any heuristic algorithm must 1) provide a feasible solution at all times and 2) be fast enough to handle reasonably large networks. The price to pay of course is that the optimality becomes a secondary consideration.

The first heuristic that we propose is based on the concept of a merit function for an allocation. We denote this function $\zeta(i, j, z)$ and it is some number that reflects how good we think is the allocation of subchannel $j$ to node $i$ with transmission mode $z$.

At iteration $k$, there is a set $\mathcal{I}$, that contains all the CPEs that are currently allocated the minimum rate value. This we call the lower bound. Initially, $\mathcal{I}$ is equal to the set of all CPEs and the bound is 0 . Because the objective function is a maxmin, in order to improve the objective, we must increase the rate of all CPEs in this set.

We make a list of all feasible $(i, j, z)$ for all CPEs $i$ in $\mathcal{I}$. We sort the list in decreasing order of the merit function and start allocating from the top of the list. Whenever an allocation $(i, j, z)$ is made, we remove from the list all the triplets with node $i$ or subchannel $j$. We keep track of the power that is being spent so that we do not exceed the power constraint. If we can raise the rate of all the CPEs in $\mathcal{I}$, then we can proceed to the next iteration. Otherwise, the algorithm stops and declares the current solution optimal.

\section{Heuristic 1}

$\lambda_{i} \quad$ current rate allocated to CPE $i$

$\mathcal{J} \quad$ a set of subchannels

$\mathcal{L} \quad$ set of candidate triplets $(i, j, z)$

$D \quad$ Set of channel allocations

$P_{t} \quad$ Total power allocated at any step in the algorithm

1) Initialization: $\mathcal{J}=$ set of all subchannels, $\mathcal{L}=$ set of all $(i, j, z), \lambda_{i}=0, D=\emptyset, P_{t}=0$

2) While $\mathcal{J} \neq \emptyset$

a) $\bar{\lambda}=\min _{i=1 \ldots N} \lambda_{i}$

b) $\mathcal{L}=\left\{(i, j, z) \mid \lambda_{i}=\bar{\lambda}, j \in \mathcal{J}, f_{i j}(z) \leq \bar{P}_{j}\right\}$

c) Sort $\mathcal{L}$ in decreasing order of $\zeta(i, j, z)$

d) While $\mathcal{L} \neq \emptyset$

i) $\left(i^{*}, j^{*}, z^{*}\right)=\mathcal{L}_{1}$

ii) If $P_{t}+f_{i^{*} j^{*}}\left(z^{*}\right) \leq \bar{P}_{\max }$ then

A) $D=D \cup \mathcal{L}_{1}$

B) $P_{t}=P_{t}+f_{i^{*} j^{*}}\left(z^{*}\right)$

C) $\lambda_{i}=\lambda_{i}+R_{z *}$

D) $\mathcal{L}=\mathcal{L}-\cup_{j, z}\left(i^{*}, j, z\right)-\cup_{i, z}\left(i, j^{*}, z\right)$

E) $\mathcal{J}=\mathcal{J}-j^{*}$

else

A) $\mathcal{L}=\mathcal{L}-\left(i^{*}, j^{*}, z^{*}\right)$

B) if $\mathcal{L}=\emptyset$ stop with the current allocation $D$ endif

endif

end while

end while

When the algorithm stops, the power constraint is met and each subchannel has been allocated at most once so that the solution is feasible. The quality of the solutions will depend on the merit function. The results presented in section $\mathrm{V}$ were obtained with a merit function of the form $\zeta(i, j, z)=$ $R_{z} /\left(\log _{2}\left(f_{i j}(z)+2\right)\right)$. This tends to allocate subchannels that have both a high rate and a low power requirement.

\section{Heuristic 2}

In this section, we propose another sub-optimal algorithm with low complexity to solve problem (1)-(4). This heuristic comprises 3 steps.

Similar to Heuristic 1, we would like to adopt a roundrobin like approach to subchannel allocation, i.e., we allocate subchannels to CPEs whose current allocated rate is the lowest. The difficulty is two-fold. First, we need to estimate the quality of each channel with respect to each CPE and we need to estimate the total rate that is given to a CPE at every step. In Heuristic 1, the first was accomplished by the merit function (which ignored power) and the second by essentially giving to each CPE the requisite power to achieve the rate dictated by the merit function until power runs out.

In this heuristic, we more explicitly account for power. One approach would be to assign each CPE, in advance, an equal amount of the total power budget. This, however, would provide excess power to users near the base station while starving users far from the base station.

Hence in our first step (presented below), we allocate power as evenly as possible across subchannels, taking into account the constraints $\bar{P}_{j}$. This is relatively fair as subchannels are not initially assigned to CPEs. In addition, it has been shown in [8] in the context of continuous rates that subchannel allocation based on a uniform power allocation can provide good performance compared to the optimal solution.

\section{Step 1: Power Allocation}

$P_{l} \quad$ remaining power

$p_{j} \quad$ power allocated to subchannel $j$

$A$ set of subchannels without power allocation

1) if $\sum_{j=1}^{M} \bar{P}_{j} \leq \bar{P}_{\max }$

a) Allocate power as $p_{j}=\bar{P}_{j}$, for $j=1,2, \ldots, M$ else

a) Initialization: $A=\{1,2, \ldots, M\}, P_{l}=\bar{P}_{\max }$

b) while $\min _{j \in A} \bar{P}_{j}<P_{l} /|A|$

i) Find $j^{*}=\operatorname{argmin}_{j \in A} \bar{P}_{j}$

ii) Allocate $p_{j^{*}}=\bar{P}_{j^{*}}{ }^{*}$

iii) Update $P_{l}=P_{l}-p_{j^{*}}, A=A-\left\{j^{*}\right\}$ end while

c) for $j=1$ to $M$ if $j \in A$ then allocate $p_{j}=P_{l} /|A|$ end for

endif

Note that $|A|$ denotes the cardinality of set $\mathrm{A}$. In fact, in this sub-algorithm we consider two cases. The first case occurs when $\sum_{j=1}^{M} \bar{P}_{j} \leq \bar{P}_{\text {max }}$ so the total power constraint at the BS is not active. Hence, we allocate power to different subchannels based on the constraints in vector $T$. Otherwise, we will allocate an amount of power equal to the corresponding power limits in vector $T$ (i.e., $p_{j}=\bar{P}_{j}$ ) for the subchannels with tight constraints (i.e., small $\bar{P}_{j}$ ) and share the rest equally among the other subchannels.

Given the power allocation in the first step, in the second step we perform subchannel allocation as in the following. Let $h_{i}\left(p_{j}\right)$ be the function which gives the maximum achievable rate on subchannel $j$ for CPE $i$ with transmit power $p_{j}$.

\section{Step 2: Subchannel Allocation}

A remaining subchannels

$\lambda_{i} \quad$ current rate allocated to CPE $i$

$C_{i}$ subchannels allocated to CPE $i$ 
1) Initialization: $C_{i}=\emptyset, \lambda_{i}=0$ for $i \in\{1,2, \ldots, N\}$, $A=\{1,2, \ldots, M\}$, check=0

2) while $A \neq \emptyset$ and check $=0$

- Find $i^{*}$ such that $i^{*}=\operatorname{argmin}_{i} \lambda_{i}$

- Find the set subchannels with maximum rate: $M=$ $\left\{j^{*} \mid h_{i^{*}}\left(p_{j^{*}}\right)=\max _{j \in A}\left\{h_{i^{*}}\left(p_{j}\right)\right\}\right\}$

- Allocate subchannel $j^{*}$ for CPE $i^{*}$, i.e., $C_{i^{*}}=C_{i^{*}}+\left\{j^{*}\right\}$, where $j^{*}=\operatorname{argmax}_{j \in M}\left\{g_{i^{*} j}\right\}$

- Update $A=A-\left\{j^{*}\right\}, \lambda_{i^{*}}=\lambda_{i^{*}}+h_{i^{*}}\left(p_{j^{*}}\right)$

- If $h_{i^{*}}\left(p_{j^{*}}\right)=0$ Set check $=1$

Set $i=i^{*}+1$ end if

end while

3) If check $=1$ and $A \neq \emptyset$

while $A \neq \emptyset$

- Allocate one arbitrary available subchannel to CPE $i$ : $C_{i}=C_{i}+\{j\}$ where $j \in A$

- Update $A=A-\left\{j^{*}\right\}$

- Move to the next CPE: $i=i+1$ if $i+1 \leq N$ or $i=1$ if $i+1>N$

end while

end if

In this sub-algorithm, we compute for each CPE, the highest feasible rate on each subchannel based on the power allocation. Based on that, we assign to the CPE having the lowest current rate (ties are broken randomly), the subchannel yielding the highest rate (among those not yet allocated) with ties broken in favor of the subchannel with the largest channel gain $g_{i j}$. In this sub-algorithm, $A$ denotes the set of remaining subchannels, $\lambda_{i}$ represents the current allocated rate and $C_{i}$ denotes the current set of subchannels allocated to CPE $i$. If at a given time, we cannot improve the rate of the CPE under consideration, then we share the remaining subchannels among the CPEs in a round-robin fashion and we terminate step 2. Otherwise, step 2 ends when $A$ becomes empty.

Once the subchannels has been assigned in step 2, there is a potential gain in recomputing the power and rate allocation (e.g., subchannels assigned to CPEs near the BS might be over-provided with power). Hence, in step 3, based on the subchannel allocation, we perform rate and power allocation for all the subchannels while respecting both the total power constraint at the BS and the power constraints from vector $T$. Let $z_{i j}$ denotes the transmission mode currently employed on subchannel $j$ for CPE $i$. Let us define $\Delta P_{i, j}=$ $\left[f_{i j}\left(z_{i j}+1\right)-f_{i j}\left(z_{i j}\right)\right] /\left[R_{z_{i j}+1}-R_{z_{i j}}\right]$ which denotes the extra transmit power per unit rate required to use the next transmission mode for CPE $i$ on subchannel $j$ (i.e., mode $z_{i j}+1$ is used instead of mode $z_{i j}$ ). We sequentially increment the transmission mode for the most power-efficient subchannel of the CPE which has the minimum rate taking into account the $\bar{P}_{j}$ and the sum power constraint.

Note: the solutions obtained at the end of step 2 and step 3 are always feasible.

\section{Step 3: Rate and Power Allocation}

$z_{i j} \quad$ current transmission mode for CPE $i$ on subchannel $j$.

$P_{t} \quad$ remaining power to allocate

$\lambda_{i} \quad$ current rate for CPE $i$

1) Initialization: $P_{t}=0, \lambda_{i}=0, z_{i j}=0$ for $i \in\{1,2, \ldots, N\}$, $j \in C_{i}$

2) for $i=1$ to $N$
- Set transmission mode one for CPE $i$ on subchannel $j^{*}$ where $j^{*}=\operatorname{argmin}_{\left\{j \in C_{i} \text { and } f_{i j}(1) \leq \bar{P}_{j}\right\}} \Delta P_{i, j}$

- Update $\lambda_{i}=R_{1}, P_{t}=P_{t}+f_{i j^{*}}(1)$, and $z_{i j^{*}}=1$ end for

3) while $P_{t}<\bar{P}_{\text {max }}$

- Find $i^{*}$ such that $i^{*}=\operatorname{argmin}_{i} \lambda_{i}$

- Increase transmission rate to the next transmission mode for CPE $i^{*}$ on subchannel $j^{*}$ where $j^{*}=$ $\operatorname{argmin}_{\left\{j \in C_{i^{*}} \text { and } f_{i^{*} j}\left(z_{i^{*} j}+1\right) \leq \bar{P}_{j}\right\}} \Delta P_{i^{*} j}$

- Update $\lambda_{i^{*}}=\lambda_{i^{*}}-R_{z_{i^{*} j^{*}}}+R_{z_{i^{*} j^{*}+1}}, P_{t}=P_{t}+$ $f_{i^{*} j^{*}}\left(z_{i^{*} j^{*}}+1\right)-f_{i^{*} j}\left(z_{i^{*} j^{*}}\right)$, and $z_{i^{*} j^{*}}=z_{i^{*} j^{*}}+1$ end while

\section{NUMERICAL RESULTS}

\section{A. Input Generation}

To test the performance of the heuristics presented above, we now describe the method by which we generated realistic scenarios yielding the vector $T$ and the $f_{i j}(z)$.

The power gain between the BS and a receiver $i$ (primary or secondary) at distance $d_{i}$ from the BS on subchannel $j$ is modeled as a combination of path loss and fading. In particular, we model the received power, $P_{R}$ by $P_{R}=g_{i j} P_{T}$ where $P_{T}$ is the transmitted power and $g_{i j}=\left|h_{i j}\right|^{2}\left(d_{0} / d_{i}\right)^{\eta}$, where $h_{i j}$ is an independent Ricean fading gain characterized by its $K$-factor, $\eta$ is the path loss exponent and $d_{0}$ is the far-field crossover distance.

We generate randomly and uniformly the positions of $N$ secondary users (i.e., the CPEs) in a disk of radius $r_{2}$ centered on the BS while $N_{p}$ primary receivers are placed uniformly and randomly in a disk of radius $r_{1}>r_{2}$ centered on the BS (see Fig. 1).

Furthermore, we assume that we randomly and uniformly assign one subchannel to each primary receiver. Denote this subchannel for primary receiver $n$ by $j_{n}$. Then, the $\bar{P}_{j}$ are taken to be the largest feasible value such that the received power from the BS to each primary user $n$ on channel $j_{n}$ is at most $N_{0}$ (the noise power). For simplicity we take $f_{i j}(z)=$ $\gamma_{z} N_{0} / g_{i j}$ though we could incorporate primary interference to secondary users in a more complex model.

In summary, this method allows us to obtain the "outputs" $\left\{f_{i j}(z)\right\}$ and the $\left\{\bar{P}_{j}\right\}$ as a function of the "inputs" $N, M$, $\bar{z},\{\gamma(z)\},\left\{R_{z}\right\}, N_{p}, K, d_{0}, \eta, N_{0}, r_{1}, r_{2}$. It is important to note that this method would generate random positions for the $N$ secondary users and the $N_{p}$ primary receivers and hence 2 tests cases with the same set of "inputs" would produce different sets of "outputs" and hence if it was not for space constraint, we should characterize a test case by $N, M, \bar{z}$, $\left\{R_{z}\right\},\left\{f_{i j}(z)\right\}$, the vector $T$ (i.e., the $\left.\left\{\bar{P}_{j}\right\}\right), \bar{P}_{\max }$ and the positions of all the receivers.

\section{B. Test Cases}

Using the method described above, all the test cases that we have generated share the following parameters. The $K$-factor is $-10 \mathrm{~dB}$ which reflects scenarios with little to no line of sight, $\eta=3, d_{0}=50 \mathrm{~m}, r_{1}=33 \mathrm{~km}, r_{2}=60 \mathrm{~km}$, and $N_{0}=-100 \mathrm{~dB}$. Thus a device at a distance of $33 \mathrm{~km}$ from the BS will see an average (neglecting fading) SNR of $15.4 \mathrm{~dB}$ if the BS uses a transmit power of 1 Watt. We use in all cases five transmission modes of rates $R_{1}=1, R_{2}=2, R_{3}=3$, $R_{4}=4$ and $R_{5}=5$ with SNR thresholds of $\gamma_{1}=10 \mathrm{~dB}$, $\gamma_{2}=14.77 \mathrm{~dB}, \gamma_{3}=18.45 \mathrm{~dB}, \gamma_{4}=21.76 \mathrm{~dB}$ and $\gamma_{5}=$ 


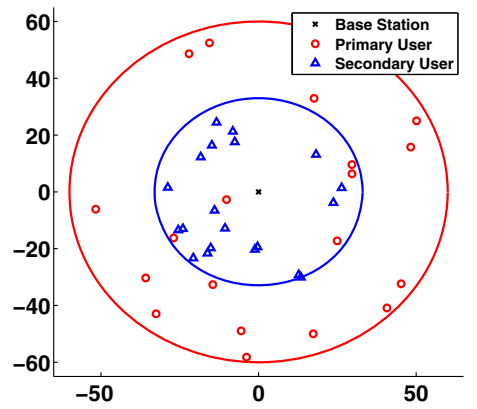

Fig. 1. Sample placement of 20 primarv and 20 secondarv users.

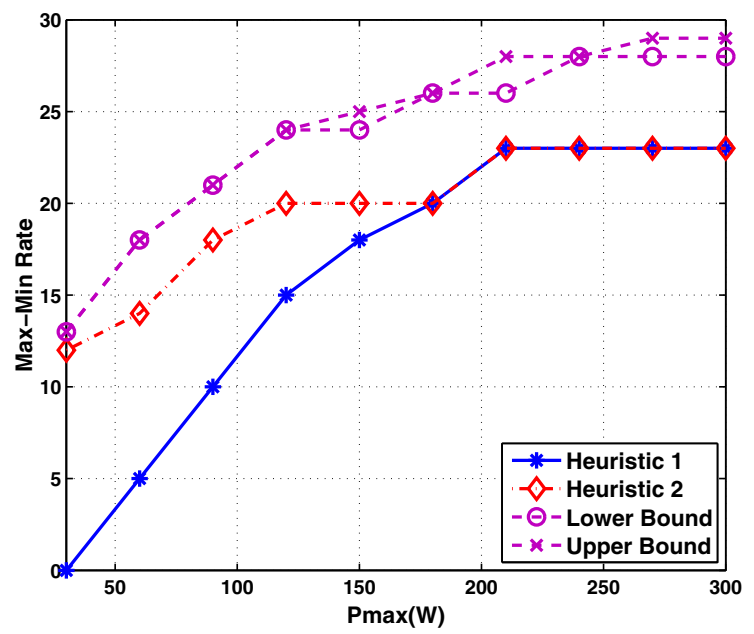

Fig. 2. Max-min rate vs. $\bar{P}_{\max }$ obtained via cplex and the 2 heuristics for a scenario with $\left(M, N, N_{p}\right)=(350,50,200)$

$24.91 \mathrm{~dB}$. The merit function used for the first heuristic is $R_{z} /\left(\log _{2}\left(f_{i j}(z)+2\right)\right)$.

\section{Results}

Figure 2 is typical of what we have seen on a very large number of scenarios. It shows the max-min rate for a scenario with 350 subchannels, 50 CPEs and 200 primary receivers obtained using cplex (limiting the running time to ten minutes) and the 2 heuristics. Most of the time, cplex was able to compute the optimal solution in less than ten minutes. However when it could not, it gave us a feasible maxmin rate that we call the lower bound as well as an upper bound. Clearly Heuristic 2 gives us much better result than Heuristic 1. Heuristic 2 also follows quite well the trend of the max-min rate as a function of $\bar{P}_{\max }$. Part of the reason for the poor performance of Heuristic 1 is that the choice of transmission ,pde on any subchannel is made without taking into consideration the remaining power budget, remaining subchannels or number of CPEs.

Figure 3 shows the relative loss in max-min rate versus $\bar{P}_{\max }$ when using Heuristic 2 instead of cplex for a scenario with 120 subchannels, $40 \mathrm{CPEs}$ and 20 primary receivers. If $Z_{c}$ denotes the optimal max-min rate computed by cplex and $Z_{h}$ by Heuristic 2 , then the relative loss is $\left(Z_{c}-Z_{h}\right) / Z_{c}$. Note that $\mathrm{cplex}$ was always able to compute the optimal solution in less than ten minutes for this scenario. For each value of $\bar{P}_{\text {max }}$, we ran 10 test cases corresponding to different values of

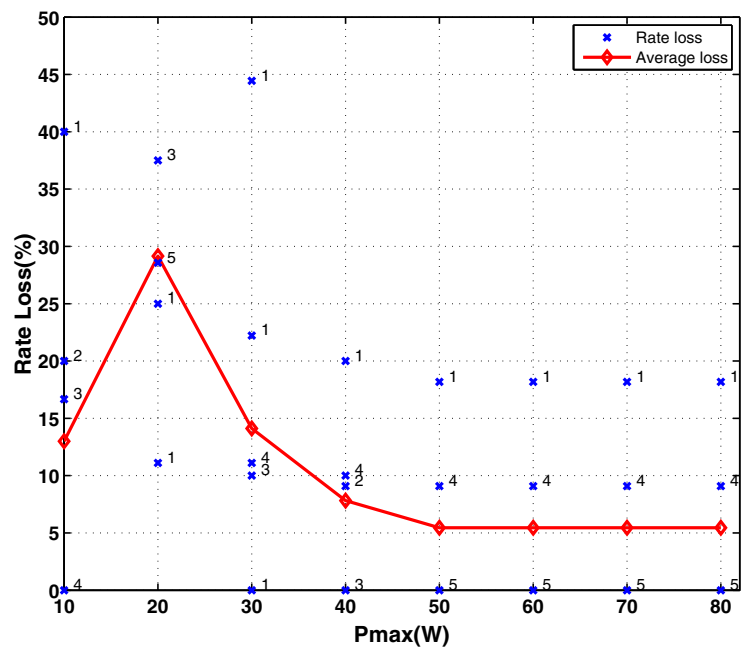

Fig. 3. Relative rate loss vs. $\bar{P}_{\max }$ of Heuristic 2 over exact solution for $\left(M, N, N_{p}\right)=(120,40,20)$

$\left\{f_{i j}(z)\right\}$ and the $\left\{\bar{P}_{j}\right\}$. The red line corresponds to the loss in rate averaged over the 10 test cases. Clearly, Heuristic 2 works relatively very well for moderate to high $\bar{P}_{\max }$ and not that well for low $\bar{P}_{\max }$.

\section{CONCLUSIONS}

In this paper, we have formulated a realistic cognitive radio resource allocation problem for the downlink of a point-tomultipoint network that allows secondary transmission provided the interference caused to primary nodes is below an interference threshold. This formulation was made possible by the vector $T$ which decoupled the spectrum sensing problem from that of resource allocation. Recognizing that this resource allocation problem had to be solved very fast, we proposed two fast heuristic algorithms that we evaluated on practical test cases. Our results show that the second heuristic which makes an initial power allocation (based on which subchannels are allocated) and then refines this power allocation performs well and is best over a wide range of test cases.

Acknowledgments: This study was supported in part by NSERC and Atria Networks LP.

\section{REFERENCES}

[1] FCC Spectrum Policy Task Force, "Report of the spectrum efficiency working group," FCC, Tech. Rep., Nov. 2002.

[2] C. Y. Wong, R. S. Cheng, and K. B. Letaief, and R. D. Murch, "Multiuser OFDM with adaptive subcarrier, bit, and power allocation," IEEE J. Sel. Areas Commun., vol. 17, no. 10, pp. 1743-1758, Oct. 1999.

[3] D. Kivanc, G. Li, and H. Liu, "Computationally efficient bandwidth allocation and power control for OFDMA," IEEE Trans. Wireless Commun., vol. 2, no. 6, pp. 1150-1158, Nov. 2003.

[4] J. Jang and K. B. Lee, "Transmit power adaptation for multiuser OFDM systems," IEEE J. Sel. Areas Commun., vol. 21, no. 2, pp. 171-178, Feb. 2003.

[5] I. Kim, I. S. Park, and Y. H. Lee, "Use of linear programming for dynamic subcarrier and bit allocation in multiuser OFDM," IEEE Trans. Veh. Technol., vol. 55, no. 4, pp. 1195-1207, July 2006.

[6] Z. Shen, J. G. Andrews, and B. L. Evans, "Adaptive resource allocation in multiuser OFDM systems with proportional rate constraints," IEEE Trans. Wireless Commun., vol. 4, no. 6, pp. 2726-2737, Nov. 2005.

[7] G. Song and Y. Li, "Cross-layer optimization for OFDM wireless networks, Part II: Algorithm development," IEEE Trans. Wireless Commun., vol. 4, no. 2, pp. 625-634, Mar. 2005.

[8] W. Rhee and J. M. Cioffi, "Increase in capacity of multiuser OFDM system using dynamic subchannel allocation," in Proc. IEEE VTC'2000. 